(c) Der/die Autor(en) 2017. Dieser Artikel ist eine Open-Access-Publikation.

\title{
Redaktion
}

F. Unglaub, Bad Rappenau

Zeichner

H. J. Schütze, Köln

\section{(1) ancina}

\section{Online teilnehmen \\ 3 Punkte sammeln auf} CME.SpringerMedizin.de

\section{Teilnahmemöglichkeiten}

Die Teilnahme an diesem zertifizierten Kurs ist für 12 Monate auf CME.SpringerMedizin.de möglich. Den genauen Teilnahmeschluss erfahren Sie dort.

Teilnehmen können Sie:

- als Abonnent dieser Fachzeitschrift, - als e.Med-Abonnent.

\section{Zertifizierung}

Diese Fortbildungseinheit ist zertifiziert von der Ärztekammer Nordrhein gemäß Kategorie $D$ und damit auch für andere Ärztekammern anerkennungsfähig. Es werden 3 Punkte vergeben.

\section{Anerkennung in Österreich}

Gemäß Diplom-Fortbildungs-

Programm (DFP) werden die auf

CME.SpringerMedizin.de erworbenen

Fortbildungspunkte von der

Österreichischen Ärztekammer 1:1 als fachspezifische Fortbildung angerechnet (§26(3) DFP Richtlinie).

\section{Kontakt}

Springer Medizin Kundenservice Tel. 08007780777

E-Mail:kundenservice@springermedizin.de

\section{CME Zertifizierte Fortbildung}

\section{Saul· K. Dresing}

Klinik für Unfallchirurgie, Orthopädie und Plastische Chirurgie, Universitätsmedizin Göttingen, Göttingen, Deutschland

\section{Behandlung der traumatisch eröffneten Bursa olecrani und der chronischen Bursitis olecrani}

\section{Zusammenfassung}

Operationsziel. Vollständige Bursektomie am Olekranon nach Wunddébridement unter Schonung von Gefäßen sowie nervalen Strukturen. Risikoadaptierte antibiotische Therapie bei septischer Bursitis und frühfunktionelle Nachbehandlung.

Indikationen. Akut traumatisch eröffnete Bursa olecrani, therapieresistente Bursitis olecrani. Kontraindikationen. Traumatische Bursaverletzung: Allgemeine Kontraindikationen gegen Anästhesie und Operation; chronische Bursitis: nicht verschließbarer Hautdefekt (plastische Deckung erforderlich), hämodynamisch instabiler Patient (z. B. bei Systemic Inflammatory Response Syndrome [SIRS] oder Sepsis), vorbestehende Hautinfektion Operationstechnik. Fächerförmige Lokalanästhesie, Wunddébridement, Darstellung der kompletten Bursa olecrani und Exzision, zusätzlich Friedrich-Wundrandexzision, Spülung, Drainage (Redondrainage, Easy-flow-Drainage, Penrose-Lasche). Abschließend Wundverschluss, Verband, elastische Wickelung und Cast-Ruhigstellung. Weiterbehandlung. Entfernung der Drainage/Lasche, Wundbeurteilung, frühfunktionelle Nachbehandlung aus der Cast-Schiene heraus, antibiotische Therapie bei septischer Bursitis über 2 Wochen, Maßnahmen nach PRICE-Schema. Entfernung der Nähte am 10.-12. postoperativen Tag. 
Ergebnisse. Von 2011-2016 behandelten wir 138 Fälle einer traumatischen Bursaeröffnung oder chronischen Bursitis olecrani, wobei 82 davon operativ versorgt werden mussten. Bei 10 Patienten war eine Vakuumtherapie („Vacuum Assisted Closure-Therapy“) mit sekundärem Wundverschluss aufgrund ausgedehnter Hautdefekte nötig; in zwei Fällen bildeten sich Fisteln, in zwei weiteren eine Wunddehiszenz. Alle Hautdefekte ließen sich ohne Lappendeckung vollständig verschließen.

\section{Schlüsselwörter}

Bursektomie · Bursitis · Ellenbogen · Olekranon · Wundheilung

\section{Lernziele}

Nach dem Studium dieses Artikels ...

- kennen Sie die anatomischen Verhältnisse der Bursa olecrani und die umgebenden Strukturen.

- können Sie eine traumatisch eröffnete Bursa olecrani in der unfallchirurgischen Wundversorgung behandeln.

- sind Sie in der Lage, eine chronische Bursitis olecrani zu therapieren.

- wissen Sie, wie Sie die Nachbehandlung beider Entitäten korrekt durchführen.

- sind Sie über mögliche Komplikationen einer Bursektomie sowie deren Behandlung informiert.

\section{Vorbemerkungen}

Die Bursa olecrani bildet sich bei Kindern im Alter von 7-10 Jahren
Die sich bei Kindern im Alter von 7-10 Jahren bildende und oberflächlich auf der Sehne des M. triceps liegende Bursa olecrani wird von zwei Schichten Synovialmembran begrenzt, kann eine Größe von $1 \times 1 \times 0,5 \mathrm{~cm}$ bis $10 \times 5 \times 2 \mathrm{~cm}$ erreichen und weist in ca. $60 \%$ der Fälle eine

\section{Treatment of traumatic lesions of the bursa olecrani and chronic bursitis olecrani}

\begin{abstract}
Objective. Complete olecranon bursectomy with debridement, protection of veins and nerves. Riskadapted antibiotic therapy and early functional aftercare.

Indications. Acute, traumatic laceration of the bursa olecrani, chronic therapy-resistant bursitis olecrani.

Contraindications. For traumatic bursa injuries: general contraindications for anesthesia and surgery; chronic bursitis: initially not closable skin defect (plastic surgery required), hemodynamically instable patient (e.g. systemic inflammatory response syndrome [SIRS] or sepsis), pre-existing skin infection.

Surgical technique. Local anesthesia beyond the lesion, careful debridement, identification and removal of the entire bursa, excision of contaminated skin, lavage, drain insertion (Redon, Easyflow, Penrose). Wound closure, elastic bandage, and splint.

Postoperative management. Elastic bandage for 2 days, followed by drain removal. Wound assessment, early functional aftercare without splint, antibiotic therapy in septic bursitis for 2 weeks, PRICE scheme. Removal of stitches after 10-12 days.

Results. Over 5 years, 138 cases of traumatic bursa lesion or chronic bursitis olecrani were treated in our clinic, 82 patients underwent surgery. Ten patients were treated with vacuum-assisted closure therapy and consecutive wound healing; fistulae occurred in two patients and in another two dehiscence developed. All of the defects could be closed without flaps.
\end{abstract}

\section{Keywords}

Bursectomy · Bursitis · Elbow · Olecranon · Wound healing 
septierte oder lobulierte Form auf [1, 2, 3, 4, 5]. Mit ca. 0,01-0,23 \% der Krankenhauseinweisungen [6, 7, 8] wird die Häufigkeit einer Bursitis olecrani („student's elbow“) sicherlich unterschätzt, da viele Patienten ambulant verbleiben. Die Hauptursache einer Bursitis olecrani liegt in der traumatischen Eröffnung [9], welche über bakterielle Einwanderung in einer septischen Bursitis münden kann, wobei der häufigste Erreger dann Staphylococcus aureus ist [10, 11, 12, 13]. Weitere Ursachen stellen körperliche Arbeit, rheumatoide Arthritis und Gichtarthritis dar [14]. Fieber sowie ein Temperaturunterschied der betroffenen Seite zur Gegenseite von $\geq 2,2{ }^{\circ} \mathrm{C}$ sind sichere Merkmale der septischen Bursitis, während lokale Überwärmung, Rötung und Schmerzen als weniger spezifisch gelten [15].

Davon zu unterscheiden ist die aseptische Bursitis, welche klinisch nicht immer einwandfrei $\mathrm{zu}$ trennen ist, sondern Analyse und kultureller Aufarbeitung der Bursaflüssigkeit bedarf [14].

Eine Umfrage im deutschen Traumanetzwerk DGU ${ }^{\circledR}$ ergab, dass bei einer akut-traumatischen Bursaeröffnung $85 \%$ der Kliniken in Deutschland eine Bursektomie vornehmen, gefolgt von Wunddébridement in $55 \%$ und Wundrandexzision in $65 \%$ der Fälle. Eine Ruhigstellung erfolgte in mehr als $60 \%$ der Kliniken, eine antibiotische Therapie nur in $45 \%$. Nichtsteroidale Antirheumatika (NSAR) wurden in $>40 \%$ verabreicht [6]. In einer US-Studie mit 1278 Patienten wurden septische Bursitiden in $100 \%$ mittels Antibiose sowie zusätzlich $47 \%$ chirurgisch behandelt [14]. Aseptische Bursaverletzungen wurden in $47 \%$ chirurgisch, in 1,9\% allein antibiotisch behandelt [14].

Bei aseptischer Bursitis werden NSAR, Ruhigstellung und Kühlung empfohlen. Prädiktoren für den Übergang in eine chronische Bursitis sind uns nicht bekannt [4]. Bei der konservativen Behandlung steht das PRICE-Schema („protection“, „rest“, „,ice“, „compression“, „elevation“) mit Immobilisierung über eine Woche im Vordergrund [16]. Steroidinjektionen über den lateralen Zugang scheinen bei sorgfältiger Abwägung der Komplikationen ihre Berechtigung zu haben [17, 18, 19, 20]. Die Punktion von Flüssigkeit mit simultaner Kortisoninjektion kann die Rezidivrate reduzieren und wird von einigen Autoren empfohlen, ist allerdings mit erhöhten Komplikationsraten (iatrogene Infektionen, Hautatrophien) behaftet [21]. Die operative Versorgung sollte aufgrund von Wundheilungsproblemen und Rezidiven [16, 22] chronischen Fällen von aseptischer Bursitis vorbehalten bleiben [23].

Die septische Bursitis, welche ein Drittel der Bursitiden ausmacht [17, 24] geht in der Regel mit Schmerzen, Rötung, Fieber oder Schüttelfrost einher und kann zumeist mit penicillinasefesten Penicillinen(z. B. Flucloxacillin) oder Cephalosporinen der 1. Generation (z. B. Cefalexin) sowie alternativ Clindamycin (bei schweren Verläufen Vancomycin und Rifampicin-Kombinationstherapie) über 10 Tage behandelt werden $[11,25]$. Neben der historischen Hauttemperaturdifferenz [26] ist das einzig sichere Verfahren zur Unterscheidung gegenüber der aseptischen Burisitis die Kulturanlage des Bursa-Aspirats [27, 28]. Bei purulentem Aspirat oder klinischem Verdacht auf eine septische Bursitis (Fieber $>38{ }^{\circ} \mathrm{C}$, laborchemische Infektkonstellation, immunsupprimierter Patient) sollte eine Antibiose erfolgen [29]. Bei ausbleibender klinischer Rekonvaleszenz steht auch hier die operative Bursektomie als Therapieoption am Ende [28], welche auch in endoskopischer Technik durchgeführt werden kann [30, 31], jedoch komplikationsbehaftet ist [22, 32, 33].

Zusammengenommen schneidet die chirurgische Therapie gegenüber der konservativen signifikant schlechter ab [14]. Eine vollständige Bursektomie, nach Möglichkeit mit Entfernung eines Olekranonsporns [34, 35], führt aber zu zufriedenstellenden Ergebnissen [7].

Im nachfolgenden Artikel wird das Vorgehen bei akut eröffneter Bursa olecrani (• Abb. 1a) vorgestellt, welches sich operativ nicht von der Bursektomie bei chronischer Bursitis (• Abb. 1b) unterscheidet. Bezüglich des Zeitpunkts der Bursektomie bei traumatischer Eröffnung werden in der Literatur Zeitspannen von $12 \mathrm{~h}$ [36], $19 \mathrm{~h}$ [37] und $72 \mathrm{~h}$ [38] angegeben; für keine Zeitgrenze liegt eine hohe Evidenz vor [39]. Bei älteren und kontaminierten Wunden wird ein großzügiges Wunddébridement durchgeführt, gefolgt von entweder verzögerter Bursektomie oder konservativem Therapieversuch. Letzter wird nach Wundspülung mit $\mathrm{NaCl} 0,9 \%$ und ggf. Polihexanid mit $10 \%$ igem Polividon-Jod-Salbenverband durchgeführt. Alternativ erfolgt die Primärnaht innerhalb eines 24-h-Intervalls. In jedem Fall werden Bursitiden zu Beginn engmaschig kontrolliert.
Hauptursache einer Bursitis olecrani liegt in der traumatischen Eröffnung

Bei aseptischer Bursitis werden NSAR, Ruhigstellung und Kühlung empfohlen

Bei einem Drittel der Bursitiden handelt es sich um eine septische Bursitis

Bei purulentem Aspirat oder klinischem Verdacht auf eine septische Bursitis sollte eine Antibiose erfolgen

Es gibt keine hohe Evidenz für eine bestimmte Zeitgrenze der Bursektomie 

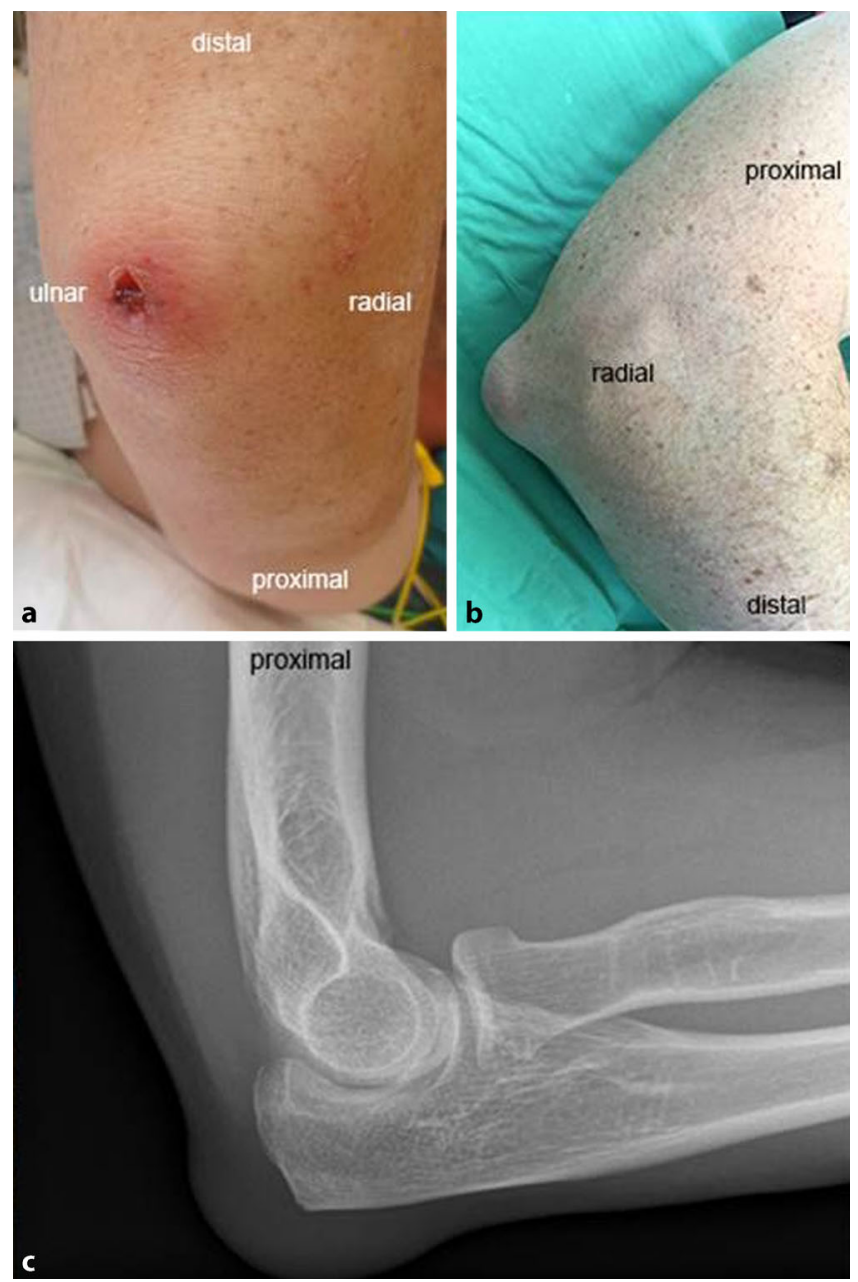

Abb. $1<$ Beispiel einer akuten Bursaeröffnung (a) und chronischen Bursitis olecrani (b). Im Röntgenbild (c) vom Patienten aus (b) zeigt sich deutlich die Schwellung der Bursa als Weichteilschatten

\section{Operationsprinzip und -ziel}

Ziel des Eingriffs ist die vollständige Bursektomie am Ellenbogen mit Wunddébridement unter Schonung umliegender Strukturen. Eine gezielte Antibiotikatherapie ist bei septischer Bursitis und/oder stark kontaminierten Wunden indiziert. Die Nachbehandlung erfolgt ggf. mit Cast-Ruhigstellung und frühfunktionell.

\section{Vorteile}

- Schnell erlernbares und durchführbares operatives Vorgehen

- Ambulante Behandlung, frühfunktionelle Nachbehandlung

- Schonung der venösen und nervalen Strukturen

- Abfluss von Synovia, Blut- und Lymphflüssigkeit durch Drainagen- oder Lascheneinlage

- Gezielte Antibiotikatherapie

- Keine Nachresektion von verbliebenen Bursaanteilen notwendig

\section{Nachteile}

- Operative Therapie

\section{Indikationen}

- Akut traumatisch eröffnete Bursa olecrani

- Chronische therapieresistente Bursitis olecrani (septisch und aseptisch) 


\section{Kontraindikationen}

\section{Bei traumatischer Bursaverletzung}

Allgemeine Kontraindikationen gegen Anästhesie und Operation

\section{Bei chronischer Bursitis}

- A priori nicht verschließbarer Hautdefekt, hier plastische Deckung erforderlich

- Hämodynamisch instabiler Patient, z. B. Systemic Inflammatory Response Syndrome (SIRS) oder Sepsis

- Vorbestehende Hautinfektion

\section{Patientenaufklärung}

- Allgemeine Operationsrisiken

- Wundinfektion

- Verletzung von Nerven, z.B. N. cutaneus antebrachii posterior und Venen

- Wundheilungsstörung, sekundärer Wundverschluss, protrahierter Heilungsverlauf

\section{Operationsvorbereitung}

- Ausführliche Anamnese mit Abklärung von Risikofaktoren: z. B. blutverdünnende Medikamente, Diabetes mellitus, Hauterkrankungen, chronische Psoriasis, Gichtarthritis, rheumatoide Arthritis, CREST-Syndrom, Niereninsuffizienz, maligne Tumoren, systemischer Lupus erythematodes, Drogenabusus i. v., langfristige Steroidmedikation oder Immuninsuffizienz.

- Tetanusimmunisierung: Überprüfung des Tetanusschutzes und bei fehlender Grundimmunisierung sowie sauberen, geringfügigen Wunden Kombinationsimpfstoff (Tdap), sonst bei tiefen und/oder verschmutzten Wunden und $<2$ Tetanusimmunisierungen oder unbekanntem Status Kombinationsimpfstoff (Tdap) in Kombination mit Tetanus-Immunglobulin (250 IE simultan). Letzterer kann bei $\geq 2$ Tetanusimmunisierungen entfallen [40].

- Klinische Untersuchung

- Röntgenaufnahme des entsprechenden Ellenbogens in zwei Ebenen

- Ggf. Anlage einer Blutsperre

\section{Instrumentarium}

- Desinfektionsmittel

- Skalpell, chirurgische Pinzette, Präparierschere, Nadelhalter, Nahtmaterial (z. B. Polypropylen 3-0)

- Einmal-Instrumenten-Set

- Spüllösung

- Lasche (z. B. Redondrainage, Penrose-Lasche, 6-mm-„,easy-flow“-Drainage; [41])

- Elastische Wickel

- Material für Stützverbände/Cast

- Bei Bedarf bipolare Antikoagulation

- Bei Bedarf Blutsperre

\section{Anästhesie und Lagerung}

- Lokalanästhesie oder Kurznarkose

- Operation in Bauch- oder Rückenlage mit abduzierter Schulter und ausgelagertem in $90^{\circ}$ flektiertem Ellenbogen, Polsterung beachten

- Klebe-/Lochtuch über der Wunde

- Ggf. Anlage einer Blutsperre 


\section{Operationstechnik}

$(\bullet$ Abb. 2, 3, 4, 5, 6)

Die Operationsschritte sind bei traumatisch eröffneter Bursa olecrani identisch mit der Bursektomie bei einer chronischen Bursitis.

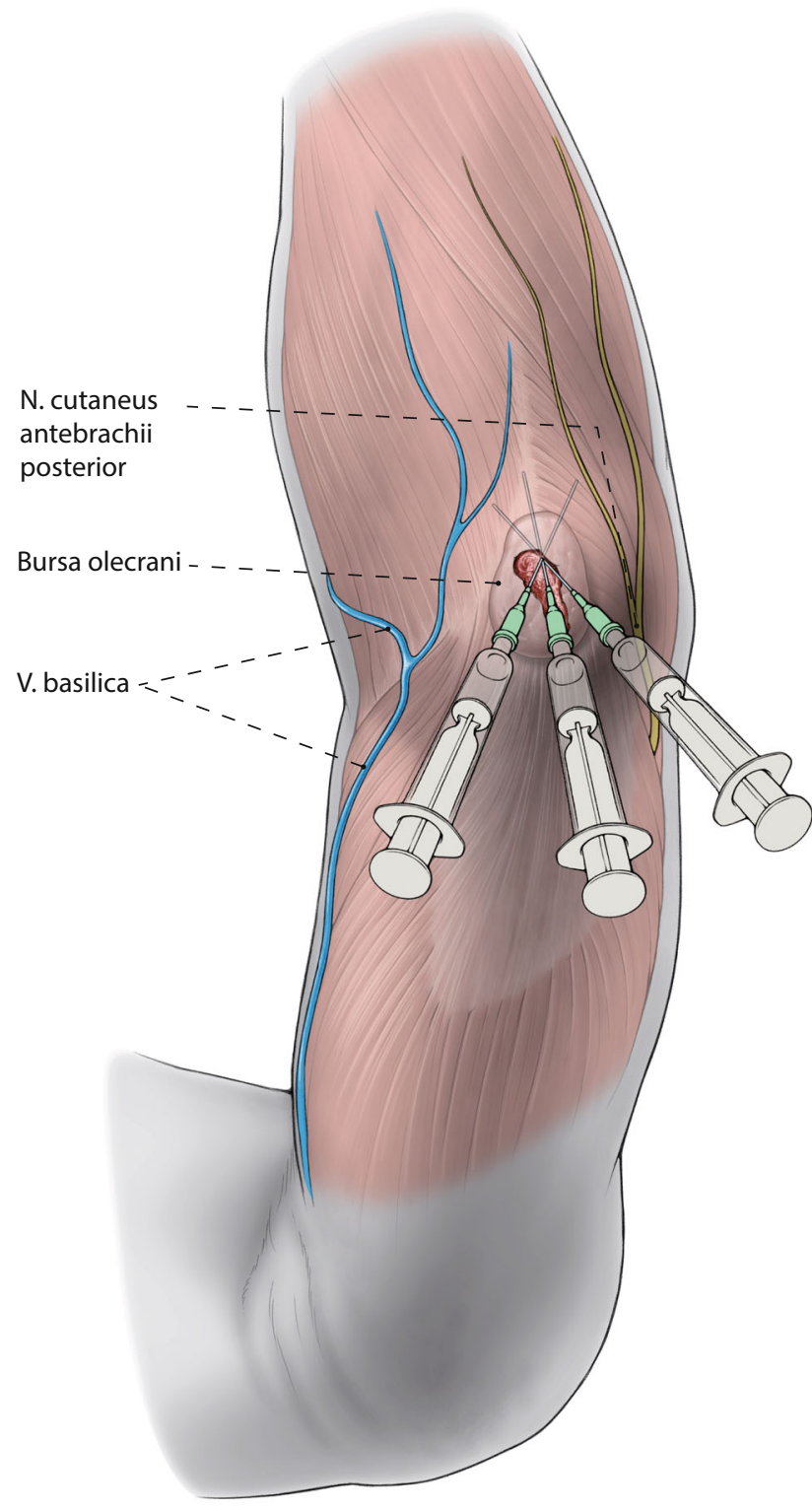

Abb. $2 \triangleleft$ Die kontinuierliche Applikation eines Lokalanästhetikums (z. B. Scandicain ${ }^{\circledR} 1 \%$ ) durch die Wundränder bei nicht verschmutzter Wunde unter die gesunde Haut wird fächerförmig durchgeführt 


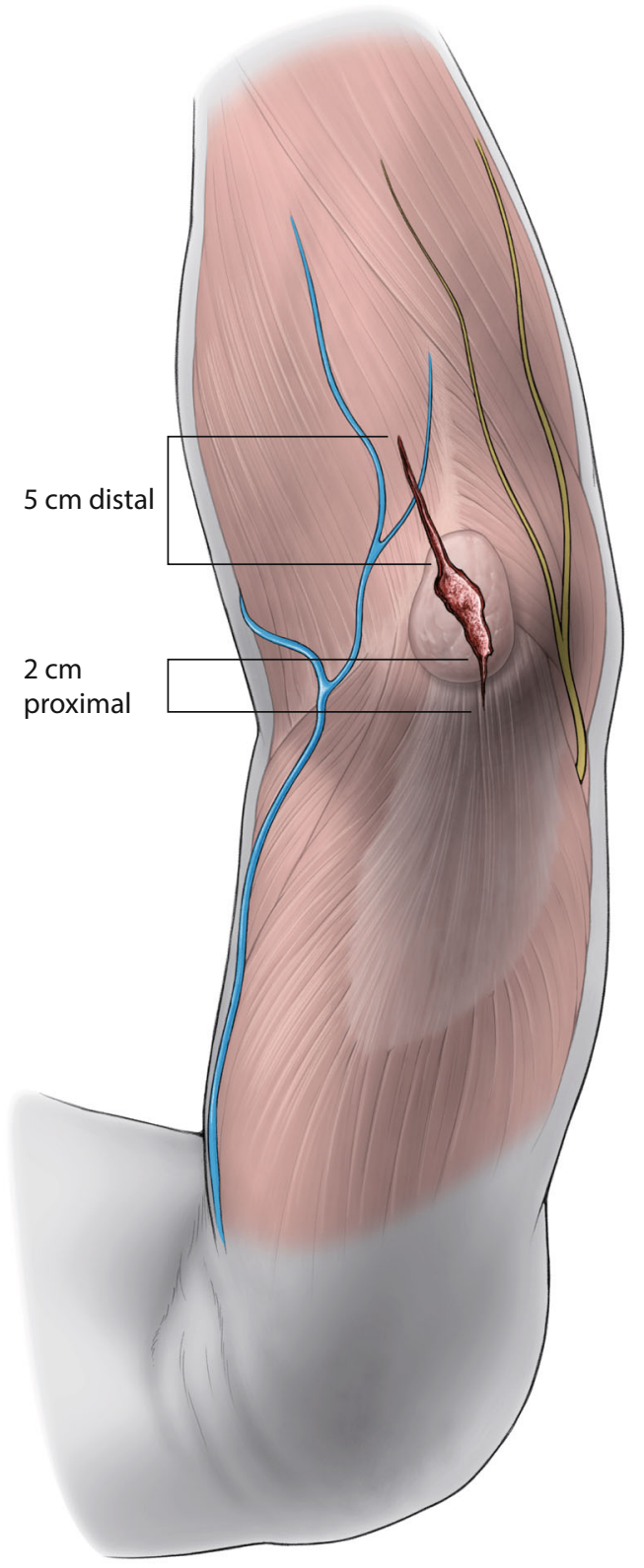

Abb. 3 A Wundrandexzision beidseits der Wunde mittels Skalpell und Schnitterweiterung $2 \mathrm{~cm}$ nach proximal des Epicondylus lateralis und maximal $5 \mathrm{~cm}$ distal über den Radiuskopf. Beachte: Der N. cutaneus antebrachii posterior (sensibler Endast des N. radialis) zieht durch den lateralen Kopf des M. triceps brachii und durchbricht die Fascia brachialis über dem lateralen suprakondylären Humerus, bevor er sich in zwei Endäste teilt

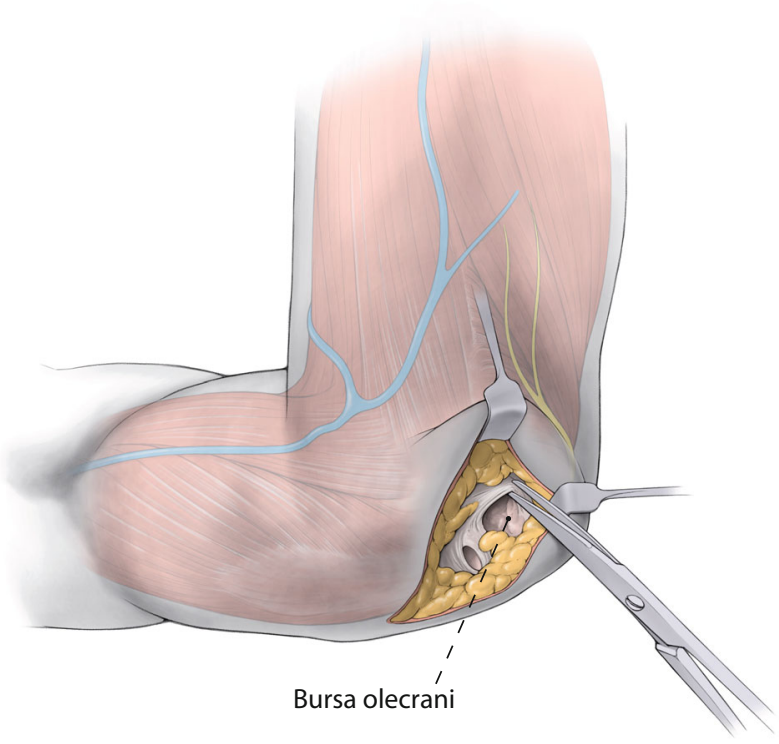

Abb. 4 \ Das Subkutangewebe wird in Längsrichtung inzidiert. Beachte: Ein sensibler Endast des N. radialis, der N. cutaneus antebrachii posterior, durchbricht die Oberarmfaszie über dem Ursprung des M. brachioradialis und teilt sich dann in Hautäste auf. Die venöse Versorgung des medialen Ellenbogens erfolgt durch Endäste der V. basilica 

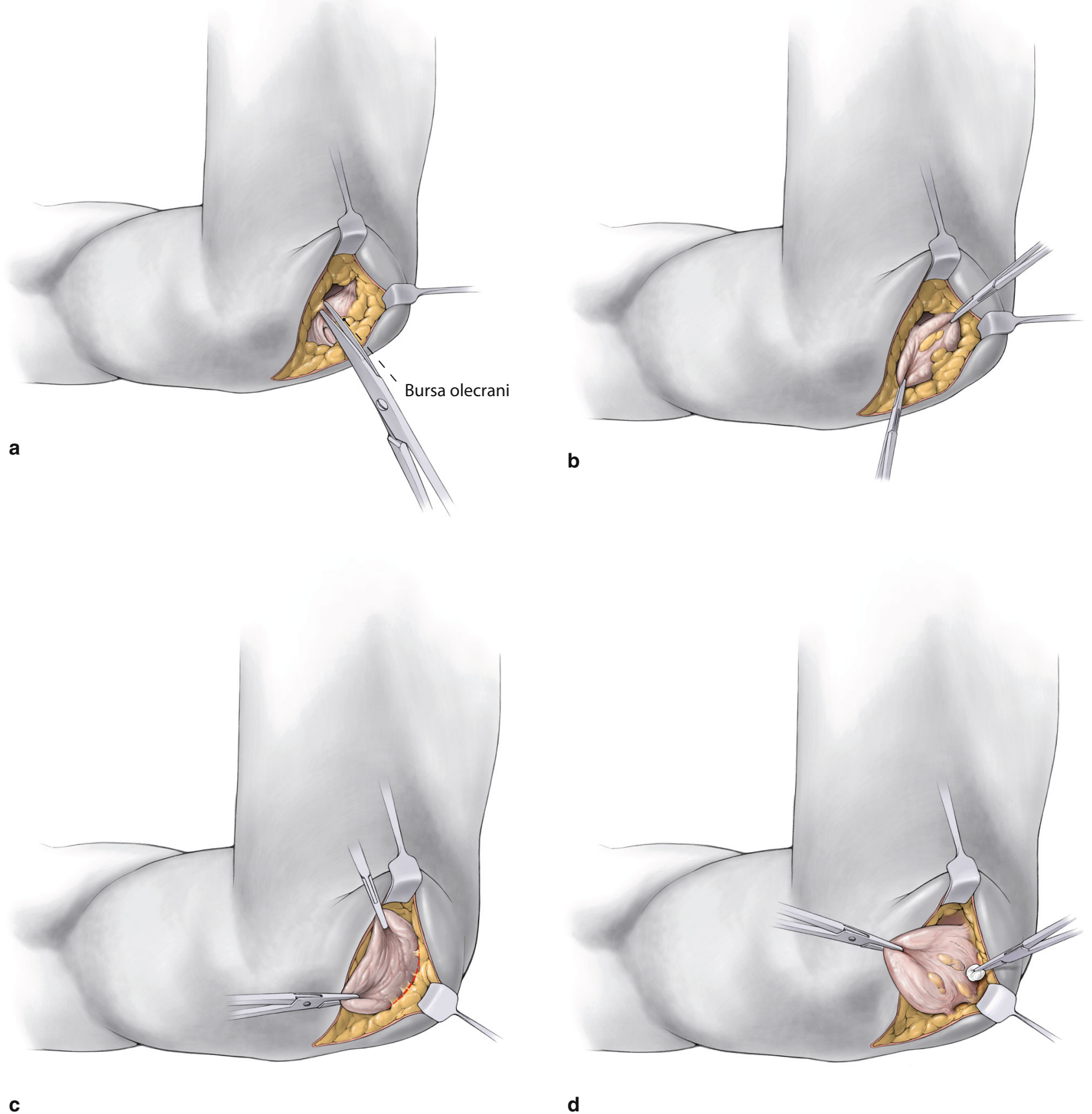

C

Abb. $5 \Delta$ Die Bursa wird mittels Präparierschere und Pinzette exploriert $(\mathbf{a}, \mathbf{b})$ und vollständig dargestellt (d), wozu dieselbe mittels Klemmchen (b) fixiert und die Grenzschicht zwischen Haut und Bursa dargestellt wird (c, d) 
a

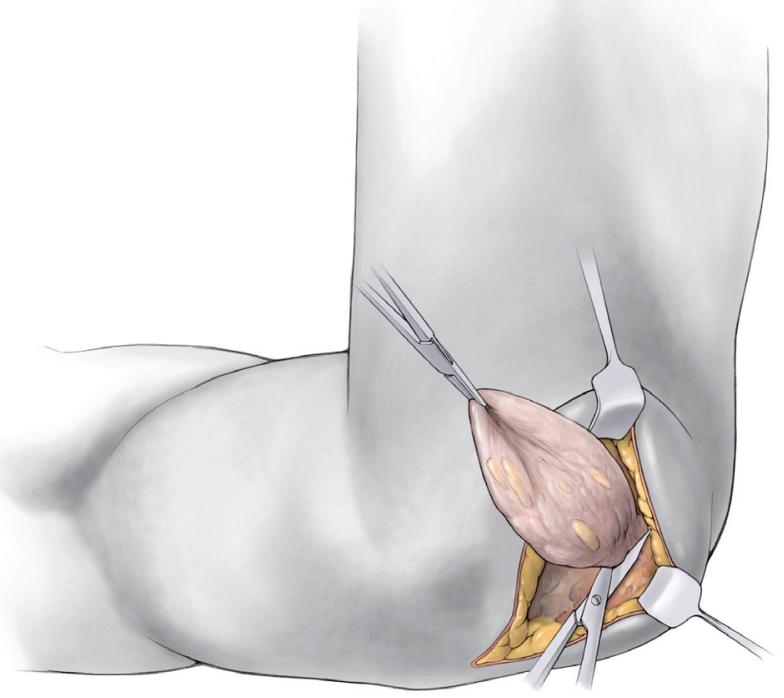

b
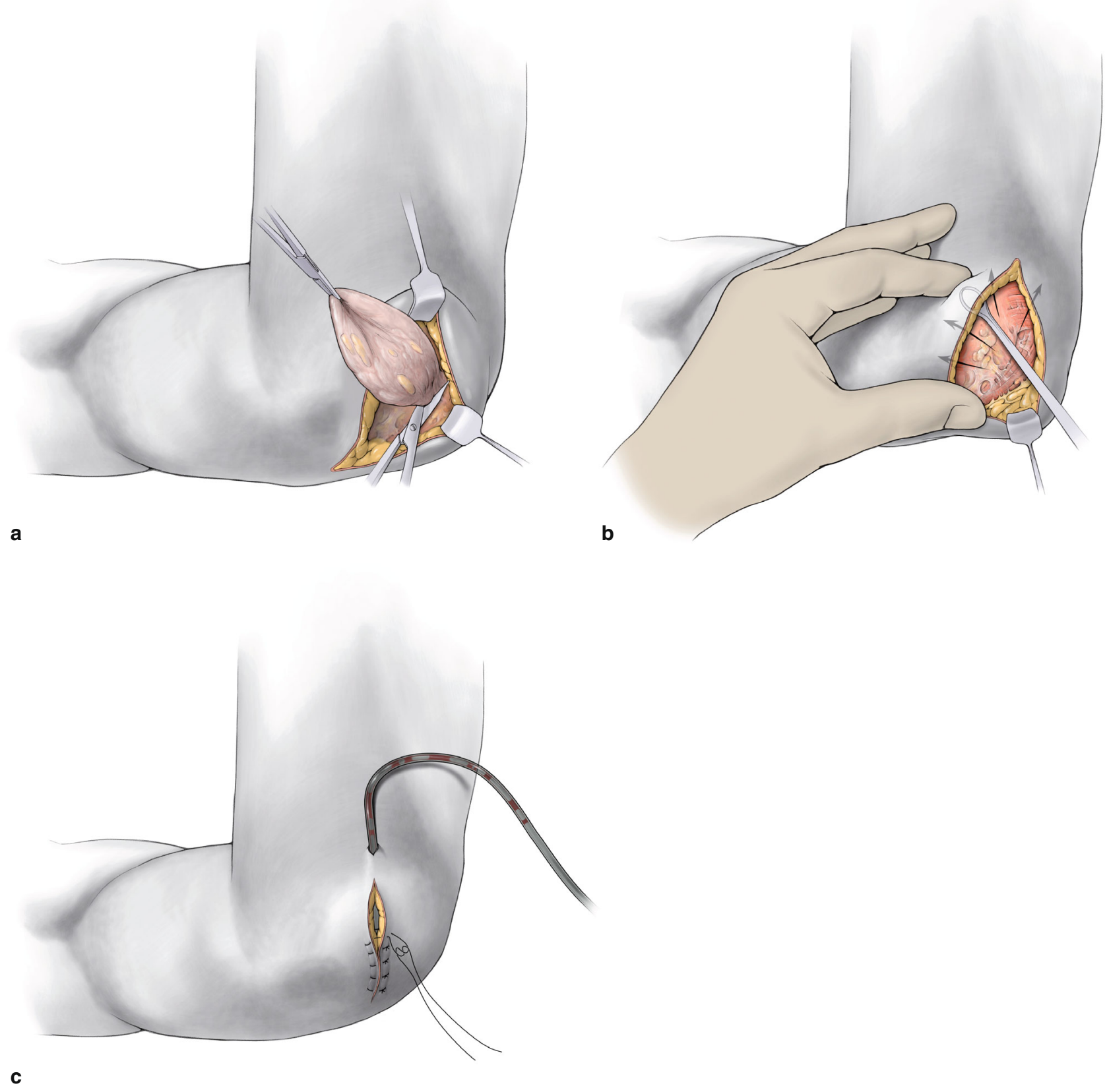

Abb. 6 ॥ Nach Darstellung von kompletter Bursa (a) samt Subkutangewebe und ausgiebigem Débridement (b) erfolgt eine vollständige Exstirpation mit nachfolgender Redondrainagen-/Lascheneinlage (c) und Hautnaht in Donati-Rückstichtechnik mit nichtresorbierbarem Fadenmaterial. Anschließend Anlage von Kompressen mit elastischer Wickelung, ggf. Schienung

\section{Postoperative Behandlung}

- Schmerzadaptierte funktionelle Nachbehandlung, ggf. $90^{\circ}$-Flexion für 2 Wochen zur Minimierung von postoperativem Hämatom [17]

- Cast-Anpassung, ggf. in $90^{\circ}$-Flexion

- Elastische Wickelung bis zur ersten Wundkontrolle

- Wundkontrolle, ggf. Drainagenentfernung am 2. postoperativen Tag

- Keine medikamentöse antithrombotische Therapie indiziert [42]

- Nahtentfernung am 10.-12. postoperativen Tag mit Therapiebeendigung 
- Bei Verdacht auf eine septische Bursitis sollten penicillinasefeste Penicilline (z. B. Oxycillin, Flucloxacillin) oder ein Cephalosporin der ersten Generation (i. v. Cefazolin, oral Cefalexin) über 2 Wochen oral verwendet werden.

- Bei systemischen Zeichen einer Infektion oder immunsupprimierten Patienten wird eine stationäre Aufnahme mit i.v.-Antibiotikum für 7-10 Tage empfohlen, mit anschließender oraler Antibiose über 2 Wochen [12, 16, 25, 29].

\section{Fehler, Gefahren, Komplikationen}

- Fehlinterpretation einer septischen als aseptische Bursitis: Antibiotikatherapie mit penicillinasefestem Penicillin (z. B. Oxycillin, Flucloxacillin) oder Cephalosporin der ersten Generation (i. v. Cefazolin, oral Cefalexin) über 2 Wochen oral, bei systemischer Infektion oder immunsupprimierten Patienten intravenöse Antibiose über 7-10 Tage mit nachfolgender oraler Antibiose über 2 Wochen [12, 16, 25, 29], Anpassung der Medikation nach Antibiogramm

- Progredienz der Bursitis mit Abszedierung, Phlegmone, SIRS bis Sepsis: Wundrevision mit Wunddébridement, ggf. Vakuum-Verband-System-Anlage, ggf. sekundärer Wundverschluss [36]; breite Antibiose, welche nach Antibiogramm deeskaliert wird; PRICE-Schema

- Wundheilungsstörung (in bis zu 30 \% der Fälle infolge einer Bursektomie, [21]): „Watchful waiting“, ggf. Revision mit Wundrandexzision und erneuter Naht, ggf. lokale, gestielte oder freie Lappenplastik $[33,43]$

- Verletzungen des N. cutaneus antebrachii posterior durch unvorsichtiges Präparieren oder erschwerte anatomische Verhältnisse durch die Bursitis selbst: Aufklärung, ggf. mikroskopische Nervennaht, konservatives Prozedere

\section{Ergebnisse}

Der häufigste Erreger war Staphylococcus aureus

\section{Alle Hautdefekte ließen sich ohne Lappendeckung schließen}

Von 2011 bis 2016 behandelten wir 138 Fälle von traumatischer Bursaeröffnung (insgesamt 71) oder Bursitis olecrani (insgesamt 67), was einem Anteil von 0,07\% unserer Patienten entsprach. Von diesen wurden 82 operativ versorgt, wobei 71 als posttraumatisch und 11 als chronisch einzustufen waren. Die Patienten mit chronischen Bursitiden waren dabei mit 54 Jahren (SD $\pm 19,4)$ etwas älter als die posttraumatischen $(40,9 \pm 23,4$ Jahre). Bei ersteren fanden sich in intraoperativen Abstrichen 9-mal Staphylococcus aureus sowie jeweils einmal Streptococcus pyogenes und Pseudomonas aeruginosa.

Insgesamt unterliefen 15 Patienten Revisionsoperationen (9 chronische, 6 posttraumatische Bursitiden), wobei VAC-Wechsel mit sekundärem Wundverschluss aufgrund ausgedehnter Hautdefekte dabei 10-mal, Fistel-/Pus-Entleerung 2-mal, eine postoperative Wunddehiszenz 2-mal und ein erneutes Sturzereignis mit Eröffnung der Naht einmal auftraten. Alle Hautdefekte ließen sich schlussendlich ohne Lappendeckung verschließen.

Im Durchschnitt waren die Patienten mit Bursitis olecrani 48,1 $\pm 20,4$ Jahre alt und zu 67,4\% männlichen Geschlechts.

Die Indikation zur Bursektomie wurde in unserem Kollektiv der traumatischen Bursaeröffnung großzügig gestellt, bei der chronischen Bursitis hingegen sehr zurückhaltend, was im Konsens mit der aktuellen Literaturlage zu werten ist $[4,7,15]$. Bei rezidivierenden Bursitiden und Versagen der konservativen Therapie sowie septischem Bild scheint uns die Bursektomie als Therapiemöglichkeit unabdingbar.

\section{Fazit für die Praxis}

- Einer von 1000 Patienten eröffnet sich seine Bursa olecrani traumatisch.

- Belastbare aktuelle Evidenz bezüglich dieser Entität findet sich spärlich.

- Bei aseptischer Bursitis ist in der Regel ein konservatives Vorgehen indiziert.

- Bei septischer Bursitis sollte eine orale 2-wöchige Antibiose verabreicht werden, unter regelmäßiger Anbindung und PRICE-Schema.

- Bei Therapieresistenz ist eine operative totale Bursektomie anzustreben, gefolgt von einer antibiotischen Therapie.

- Der Fadenzug erfolgt am 10.-12. postoperativen Tag. 
- Komplikationen sind vor allem das Übersehen einer septischen Bursitis und Wundheilungsstörungen.

\section{Korrespondenzadresse}

\section{Saul}

Klinik für Unfallchirurgie, Orthopädie und Plastische Chirurgie, Universitätsmedizin Göttingen

Robert-Koch-Str. 40, 37075 Göttingen, Deutschland

Dominik.Saul@med.uni-goettingen.de

\section{Einhaltung ethischer Richtlinien}

Interessenkonflikt. D. Saul und K. Dresing geben an, dass kein Interessenkonflikt besteht.

Dieser Beitrag beinhaltet keine von den Autoren durchgeführten Studien an Menschen oder Tieren.

Open Access Dieser Artikel wird unter der Creative Commons Namensnennung 4.0 International Lizenz (http://creativecommons. org/licenses/by/4.0/deed.de) veröffentlicht, welche die Nutzung, Vervielfältigung, Bearbeitung, Verbreitung und Wiedergabe in jeglichem Medium und Format erlaubt, sofern Sie den/die ursprünglichen Autor(en) und die Quelle ordnungsgemäßnennen, einen Link zur Creative Commons Lizenz beifügen und angeben, ob Änderungen vorgenommen wurden.

\section{Literatur}

1. Chen J, Alk D, Eventov I, Wientroub $S$ (1987) Development of the olecranon bursa. An anatomic cadaver study. Acta Orthop Scand 58(4):408-409

2. Floemer F, Morrison WB, Bongartz G, Ledermann HP (2004) MRI characteristics of olecranon bursitis. AJRAm JRoentgenol 183(1):29-34. doi:10.2214/ajr.183.1.1830029

3. Quayle JB, Robinson MP (1978) A useful procedure in the treatment of chronic olecranon bursitis. Injury 9(4):299-302

4. Reilly D, Kamineni S (2016) Olecranon bursitis. JShoulder Elbow Surg 25(1):158-167. doi:10.1016/j.jse. 2015.08.032

5. Weston WJ (1970) The olecranon bursa. Australas Radiol 14(3):323-324

6. Baumbach SF, Domaszewski $F$ Wyen H, Kalcher K, Mutschler W, Kanz K-G (2013) Evaluation of the current treatment concepts in Germany, Austria and Switzerland for acute traumatic lesions to the prepatellar and olecranon bursa. Injury 44(11):1423-1427. doi:10. 1016/j.injury.2012.08.008

7. Del Buono A, Franceschi F, Palumbo A, Denaro V, Maffulli N (2012) Diagnosis and management of olecranon bursitis. Surgeon 10(5):297-300. doi:10.1016/j. surge.2012.02.002

8. McAfee JH, Smith DL (1988) Olecranon and prepatellar bursitis. Diagnosis and treatment. West J Med 149(5):607-610

9. Garcia-Porrua C, Gonzalez-Gay MA, Ibanez D, Garcia-Pais MJ (1999) The clinical spectrum of severe septic bursitis in northwestern
Spain: a 10 year study. J Rheumatol 26(3):663-667

10. Cea-Pereiro JC, Garcia-Meijide J, Mera-Varela A, Gomez-Reino JJ (2001) A comparison between septic bursitis caused by Staphylococcus aureus and those caused by other organisms. Clin Rheumatol 20(1):10-14

11. Laupland KB, Davies HD (2001) Olecranon septic bursitis managed in an ambulatory setting. The Calgary Home Parenteral Therapy Program Study Group. Clinical and investigative medicine. Clin Invest Med 24(4):171-178

12. Perez $C$, Huttner $A$, Assal $M$ et al (2010) Infectious olecranon and patellar bursitis: Short-course adjuvant antibiotic therapy is not a risk factor for recurrence in adult hospitalized patients. J Antimicrob Chemother 65(5):1008-1014. doi:10.1093/jac/dkq043

13. Wasserzug O, Balicer RD, Boxman J, Klement E, Ambar R, Zimhony 0 (2011) A cluster of septic olecranon bursitis in association with infantry training. Mil Med 176(1):122-124

14. Sayegh ET, Strauch RJ (2014) Treatment of olecranon bursitis: A systematic review. Arch Orthop Trauma Surg 134(11):1517-1536. doi:10.1007/s00402-014-2088-3

15. Schnetzke M, Studier-Fischer S, Kneser U, Grützner P-A, Guehring T (2016) Bursitis olecrani. Obere Extremität 11(2):88-95. doi:10. 1007/s11678-016-0348-2

16. Baumbach SF, Lobo CM, Badyine I, Mutschler W, Kanz K-G (2014) Prepatellar and olecranon bursitis: literature review and development of a treatment algorithm. Arch Or- thop Trauma Surg 134(3):359-370 doi:10.1007/s00402-013-1882-7

17. Aaron DL, Patel A, Kayiaros S, Calfee R (2011) Four common types of bursitis: Diagnosis and management. J Am Acad Orthop Surg 19(6):359-367

18. Lockman L (2010) Treating nonseptic olecranon bursitis: A 3-step technique. Can Fam Physician 56(11):1157

19. Rinkel WD, Schreuders TAR, Koes BW, Huisstede BMA (2013) Current evidence for effectiveness of interventions for cubital tunnel syndrome, radial tunnel syndrome, instability, or bursitis of the elbow: a systematic review. Clin J Pain 29(12):1087-1096. doi:10.1097/ AJP.0b013e31828b8e7d

20. Smith DL, McAfee JH, Lucas LM, Kumar KL, Romney DM (1989) Treatment of nonseptic olecranon bursitis. A controlled, blinded prospective trial. Arch Intern Med 149(11):2527-2530

21. Morrey BF, Sanchez-Sotelo J (Hrsg) (2009) The elbow and its disorders, 4. Aufl. Saunders/Elsevier, Philadelphia, PA

22. Degreef I, de Smet L (2006) Complications following resection of the olecranon bursa. Acta Orthop Belg 72(4):400-403

23. McFarland EG, Mamanee $P$, Queale WS, Cosgarea AJ (2000) Olecranon and prepatellar bursitis: treating acute, chronic, and inflamed. Phys Sportsmed 28(3):40-52. doi:10. 3810/psm.2000.03.773

24. Stell IM (1996) Septic and nonseptic olecranon bursitis in the accident and emergency department - an approach to management. 
EmergMed J13(5):351-353.doi:10. 1136/emj.13.5.351

25. Martinez-Taboada VM, Cabeza R Cacho PM, Blanco R, RodriguezValverde V (2009) Cloxacillin-based therapy in severe septic bursitis: retrospective study of 82 cases. Joint Bone Spine 76(6):665-669. doi:10.1016/j.jbspin.2009.04.003

26. Smith DL, McAfee JH, Lucas LM, Kumar KL, Romney DM (1989) Septic and nonseptic olecranon bursitis. Utility of the surface temperature probe in the early differentiation of septic and nonseptic cases. Arch Intern Med 149(7):1581-1585

27. Wasserman AR, Melville LD, Birkhahn RH (2009) Septic bursitis: A case report and primer for the emergency clinician. J Emerg Med 37(3):269-272. doi:10.1016/j. jemermed.2007.03.005

28. Zimmermann B, Mikolich DJ, Ho G (1995) Septic bursitis. Semin Arthritis Rheum 24(6):391-410. doi:10 1016/50049-0172(95)80008-5

29. Im Stell (1999) Management of acute bursitis: outcome study of a structured approach. J R Soc Med 92(10):516-521

30. Ogilvie-Harris DJ, Gilbart M (2000) Endoscopic bursal resection: the olecranon bursa and prepatellar bursa. Arthroscopy 16(3):249-253

31. Tu CG, McGuire DT, Morse LP, Bain GI (2013) Olecranon extrabursal endoscopic bursectomy. Tech Hand Up Extrem Surg 17(3):173-175. doi:10.1097/BTH. ob013e31829c0535
32. Blackwell JR, Hay BA, Bolt AM Hay SM (2014) Olecranon bursitis: a systematic overview. Shoulder Elbow 6(3):182-190. doi:10.1177/ 1758573214532787

33. Damert HG, Altmann S, Schneider W (2009) Soft-tissue defects following olecranon bursitis. Treatment options for closure (Weichteildefekte nach komplizierter Bursitis olecrani : Therapieoptionen zur Defektdeckung am Ellenbogen). Chirurg 80(5):448-454. doi:10. 1007/s00104-008-1586-7

34. Baker CL, Cummings PD (1998) Arthroscopic managementof miscellaneous elbow disorders. Oper Tech Sports Med 6(1):16-21. doi:10. 1016/S1060-1872(98)80033-6

35. Stewart NJ, Manzanares JB, Morrey BF (1997) Surgical treatment of aseptic olecranon bursitis. J ShoulderElbow Surg 6(1):49-54

36. Kaiser P, Schmidle G, Raas C, Blauth M (2015) Treatment concept for a traumatic lesion of the prepatellar bursa (Behandlung der traumatisch eroffneten Bursa praepatellaris). Oper Orthop Traumatol 27(5):427-436. doi:10.1007/ s00064-015-0414-8

37. Berk WA, Osbourne DD, Taylor DD (1988) Evaluation of the 'golden period' for wound repair: 204 cases from a Third World emergency department. Ann Emerg Med 17(5):496-500

38. Bongartz W, Lindner HO, Schumpelick V (1988) Primary suture of older and contaminated wounds. A prospective clinical study (Zum primaren Verschluss veralteterbzw. kontaminierter Wunden. Eine prospektive klinische Studie). Chirurg 59(11):767-770

39. van den Baar MT, van der Palen J, Vroon MI, Bertelink P, Hendrix R (2010) Is time to closure a factor in the occurrence of infection in traumatic wounds? A prospective cohort study in a Dutch level 1 trauma centre. Emerg Med J 27(7):540-543. doi:10.1136/emj. 2009.075846

40. STIKO (2015) Epidemiologisches Bulletin Nr. 34. Empfehlungen der Ständigen Impfkommission (STIKO) am Robert Koch-Institut/ Stand: August 2015. Ständige Impfkommission am Robert KochInstitut, Berlin

41. Berger A, Schmit-Neuerburg KP, Henkel R et al (2013) Tscherne Unfallchirurgie: Teil 1: Ellenbogen, Unterarm; Teil 2: Hand. Springer Berlin, Heidelberg

42. Arbeitsgemeinschaft der Wissenschaftlichen Medizinischen Fachgesellschaften (2015) S3-Leitlinie Prophylaxe der venösen Thromboembolie (VTE). http://www.awmf. org/uploads/tx_szleitlinien/003001I S3 VTE-Prophylaxe 201512.pdf,Zugriffsdatum:02.02.2017, 20:16. Zugegriffen: 2. Feb 2017

43. Hollevoet N, Vanhove W, Verdonk R (2010) Treatment of chronic wounds at the olecranon. Acta Orthop Belg 76(1):22-26 


\section{CME-Fragebogen}

Teilnahme am zertifizierten Kurs auf CME.SpringerMedizin.de

- Der Teilnahmezeitraum beträgt 12 Monate, den Teilnahmeschluss finden Sie online beim CME-Kurs.

- Fragen und Antworten werden in zufälliger Reihenfolge zusammengestellt.

- Pro Frage ist jeweils nur eine Antwort zutreffend.

- Für eine erfolgreiche Teilnahme müssen 70\% der Fragen richtig beantwortet werden.

? Die Sehne welchen Muskels liegt in anatomischer Nachbarschaft zur Bursa olecrani?

O. biceps brachii

O M. brachioradialis

O M. triceps brachii

O M. pronator teres

O M. coracobrachialis

Wie häufig findet sich eine septierte oder lobulierte Bursa olecrani?

○ $20 \%$

○ $40 \%$

○ $60 \%$

○ $70 \%$

○ $80 \%$

Ein 55-jähriger Mann stellt sich mit geröteter sowie überwärmter Bursa, Fieber und laborchemischer Infektkonstellation bei Ihnen vor. Welches ist der häufigste Erreger dieser septischen Bursitis?

a) Staphylococcus aureus

b) Streptococcus pneumoniae

O c) Escherichia coli

d) Mycobacterium tuberculosis

e) Saccharomyces cerevisiae

?as ist nicht Teil des PRICE-Schemas? Prevention (Vorbeugung)

Ice (Kühlung)

○ Rest (Ruhe)

Elevation (erhöhte Lagerung)

Kompression (Compression)
Welcher Nerv bedarf besonderer Beachtung bei der operativen Bursektomie?

O N. cutaneus antebrachii posterior

O N. femoralis

O N. medianus

O N. trochlearis

O N. thoracicus longus

? In Ihrer Ambulanz stellt sich eine 40jährige Frau mit einer seit 3 Tagen bestehenden asymptomatischen Schwellung der rechten Bursa olecrani vor. Welches Vorgehen empfehlen Sie?

O Intravenöse Antibiose mit $2 \mathrm{~g}$ Flucloxacillin

O Sofortige operative Entfernung der Bursa

MRT des rechten Ellenbogens

O Skelettszintigraphie des rechten Ellenbogens

Konservative Therapie mit Kühlung und Ruhigstellung

? Bei septischer Bursitis wird welche Antibiose im ambulanten Rahmen empfohlen?

O Vancomycin

Doxycyclin

○ Flucloxacillin

○ Piperacillin/Tazobactam

O Linezolid ?as ist im Rahmen einer septischen Bursitis am unwahrscheinlichsten?

O Schmerzen

Rötung des Ellenbogens

O Fieber

O Diarrhoe

Schüttelfrost

? Was ist nicht Teil des postoperativen Therapieregimes nach Bursektomie am Ellenbogen?

O Antithrombotische Therapie

O Cast-Ruhigstellung

○ Regelmäßige Wundkontrolle

O Schmerzadaptierte funktionelle Nachbehandlung

O Bei septischer Bursitis orale Antibiose

Was ist keine Therapiemöglichkeit nach komplizierter Bursitis?

○ "Watchful waiting"

O Fasziokutaner Transpositionslappen

O PRICE-Schema

Intravenöse Antibiose über 7-10 Tage

○ Anlage eines Fixateur externe 